\title{
Alcohol intake stimulates epithelial proliferation in an authentic model of the human breast
}

Anke Schennink ${ }^{1}$, Josephine F. Trott ${ }^{1}$, Grace E. Berryhill ${ }^{1}$, Caitlin E. Donovan ${ }^{1}$, Rodrigo

Manjarin $^{1,2}$, Monica K. VanKlompenberg ${ }^{1,3}$, Ashley R. Rowson-Hodel ${ }^{1,4}$, Michelle-Yvette Osorio Luis $^{1}$ and Russell C. Hovey ${ }^{1}$

${ }^{1}$ Department of Animal Science, University of California Davis, One Shields Avenue, Davis CA, USA

${ }^{2}$ Current Address: USDA/ARS Children's Nutrition Research Center, Baylor College of Medicine, 1100 Bates Street, Room 9022, Houston, TX

${ }^{3}$ Current Address: Indiana University School of Medicine, A103 Harper Hall, 1234 Notre Dame Ave, South Bend IN, USA

${ }^{4}$ Current Address: Department of Biochemistry and Molecular Medicine, University of California Davis School of Medicine, Sacramento, CA, USA

To Whom Correspondence Should Be Addressed

Dr. R.C Hovey, Department of Animal Science, University of California Davis, One Shields Avenue, Davis CA, 95616 USA. E-mail: rchovey@ucdavis.edu. Ph (530) 752 1682. Fax (530) 7520175

Keywords: alcohol, mammary gland, terminal ductal lobular unit

\begin{abstract}
Abbreviations
DAB, 3,3'-diaminobenzidine tetrahydrochloride; PH3, phosphorylated histone H3; P-Stat5, phosphorylated signal transducer and activator of transcription-5; MG, mammary gland; TDLU, terminal ductal lobular unit; BAC, blood alcohol concentration; IGF-I, insulin-like growth factor-I; IGFBP3, insulin-like growth factor binding protein-3; PR, progesterone receptor.
\end{abstract}




\section{ABSTRACT}

The voluntary consumption of alcohol by humans is a modifiable lifestyle factor that has consistently been linked to a woman's risk of developing breast cancer. We have used an animal model that closely recapitulates breast development in humans to study the effect of alcohol intake on breast growth and morphology. Pubertal female pigs were fed alcohol for 4-5 weeks at $19-21 \%$ of total caloric intake, which led to average blood alcohol contents of 115-130 mg/dL. Alongside increased liver mass, alcohol intake promoted the formation of distended ductules within lobular units in association with increased epithelial proliferation. Alcohol consumption also increased phosphorylation of the transcription factor STAT5 in the mammary epithelium, but did not lead to any evidence of precocious lactogenesis. In conclusion, feeding alcohol to female pigs having a similar physiology and mammary gland morphology to humans during a reproductive state equivalent to human adolescence leads to increased mammary gland proliferation and development of atypical lobular structures. These changes may phenocopy how alcohol intake increases the risk for developing breast cancer in humans. 


\section{INTRODUCTION}

An association between alcohol intake and neoplasia of the breast was first reported in 1977 [1], and has since been reinforced in a number of epidemiological studies [2-4] including in a metaanalysis of ninety-eight studies [5]. The relationship between alcohol intake and breast, oral or digestive tract cancers is specific in that there is no increased risk for other cancers including ovarian, endometrial, thyroid or renal cancers [2]. Indeed, an estimated $4 \%$ of the breast cancers diagnosed in developed countries can be attributed to alcohol intake [4].

The risk for developing breast cancer is increased even by moderate alcohol intake (10 g/day) [6], and increases by 7-12\% for every additional drink imbibed per day [2, 4, 5, 7]. At least 50\% of women between 18-44 years of age regularly consume alcohol, and of these, 17\% binge drink, which is defined as consuming alcohol to a BAC in excess of $80 \mathrm{mg} / \mathrm{dL}$ [8]. For women, binge drinking typically involves consuming 4 or more drinks within approximately 2 hours [8]. In younger women, 43\% of high school girls report alcohol use and, of these, 58\% binge-drink [9]. A recent prospective study revealed that teenage girls who consumed one drink per day had a 1.5-fold increased risk for developing benign breast disease compared to those who drank less than once/week, while girls who consumed alcohol 6-7 days/week had a 5.5-fold higher risk for developing benign breast disease [3]. Along these same lines, adults who reported alcohol consumption between ages 18 and 22 had higher rates of developing proliferative benign breast disease [10]. Given that the breast undergoes substantial development during adolescence in response to a changing endocrine environment [11], any increase in epithelial proliferation or exposure to transforming events during this window may increase the risk for developing breast cancer later in life $[12,13]$. 
The mammary glands (MG) of pigs represent an excellent model for the human breast given similarities in the histological and morphological features of the epithelium and stroma $[14,15]$. At the same time, pigs are genetically more similar to humans than mice [16] and have a similar physiology to humans [17]. Moreover, pigs voluntarily consume alcohol unlike many laboratory species [18], making them an ideal model to investigate the consequences of alcohol exposure on MG development.

Herein we examined the effect of alcohol intake on various parameters of MG histology, morphology and proliferation in pigs, where we hypothesized that alcohol consumption would stimulate precocious MG development consistent with a mechanism for increasing breast cancer risk in women. Our data reveal that voluntary alcohol intake increased the proliferation of mammary epithelial cells alongside increased phosphorylation of signal transducer and activator 5 (STAT5) and disruption of epithelial histomorphogenesis.

\section{METHODS}

Animals and tissues

Experiments were performed in accordance with the NIH Guide for The Care and Use of Laboratory Animals, as approved by the UC Davis Institutional Animal Care and Use Committee. Hampshire x Yorkshire (F1) nulliparous pubertal 6-month old female pigs were housed under standard management conditions at the University of California, Davis with free access to water, and were fed a corn and soybean mash $(14.4 \%$ protein, $0.78 \% \mathrm{w} / \mathrm{w}$ lysine, $3174.6 \mathrm{kcal} / \mathrm{kg}$ metabolisable energy). Animals were fed twice-daily, at 8am and 8pm.

Experiments were conducted in two blocks. Block 1 was a pilot experiment using two animals ( $\mathrm{n}=1$ control and alcohol-fed; average bodyweight $91 \pm 6 \mathrm{~kg}$ ), where eight animals were used in block 2 ( $\mathrm{n}=4$ per treatment; $124 \pm 2 \mathrm{~kg}$ ). In block one, females underwent a one-week adaptation 
period during which feed and water volume were increased each day. Ethanol [3.99 kcal/g metabolizable energy, the same as soluble carbohydrate ; 19, 20] was introduced on day 4 at 140 $\mathrm{mL} / \mathrm{d}$. Feed was increased for both animals on day 5 to $2.27 \mathrm{~kg} / \mathrm{d}$ with $1.6 \mathrm{~L}$ water ( \pm ethanol) which was then maintained for the duration of the experiment. Ethanol was increased daily to a maximum dose on day $8(550 \mathrm{~mL} / \mathrm{d})$, at which point the alcohol-fed female received $19 \%$ of her total caloric intake as ethanol. Pigs were weighed on days 1 and 36 of the study. For females in block 2, feed supplied was increased daily during the adaptation period to $2.27 \mathrm{~kg} / \mathrm{d}$ with water content increasing to $1.6 \mathrm{~L}$ on day 4 . Control-fed females were maintained on this level of feed for the duration of the study. Ethanol was first introduced on day $4(50 \mathrm{~mL} / \mathrm{d})$ then increased daily to a maximum dose on day 10 (600 mL ethanol/d). On day 12 alcohol-fed pigs had their feed reduced to $1.8 \mathrm{~kg} /$ day mash with $19-21 \%$ of their total caloric intake as ethanol (460-500 $\mathrm{mL} / \mathrm{d}$ ) in a final volume of $1.6 \mathrm{~L} / \mathrm{d}$. Females were weighed weekly. Stage of estrous was checked using a boar twice during the treatment period and confirmed that all females underwent regular estrous cyclicity. Pigs were euthanized on day 36 (block 1), or day 46 or 47 (block 2) in a USDA-inspected facility by electrocution followed by exsanguination. Liver, kidneys, heart, uterus and MG were collected and weighed at necropsy, samples were snap frozen in liquid nitrogen and stored at $-80^{\circ} \mathrm{C}$ or fixed in $4 \%$ paraformaldehyde (block 1) or $10 \%$ formalin (block 2).

\section{Blood Alcohol Concentration (BAC)}

Blood for BAC measurement was collected from pigs by jugular venipuncture into Vacutainer tubes containing sodium fluoride and potassium oxalate twice (days 17 and 35) during block 1 , and every 2-7 d immediately following the morning feeding during block 2. Plasma was separated by centrifugation and stored in tightly closed tubes on ice prior to BAC analysis at the 
UC Davis Medical Center Clinical laboratory (Sacramento, CA) using an enzymatic rate method on a Beckman Coulter DxC automated chemistry analyzer.

\section{Mammary gland assessments}

Fixed tissues obtained from three MG, distributed across the entire mammary chain in each pig, were prepared as whole mounts, without acetone defatting, as described [14, 21]. Carmine alum stained semi-thick sections ( $\mathrm{n}=3$ per MG) were mounted in Permount (Thermo Fisher Scientific, Hampton, New Hampshire, USA) and imaged with a dissecting microscope (Olympus SZX16, Shinjuku, Tokyo, Japan).

Sections (4-5 $\mu \mathrm{m})$ of paraffin-embedded tissue were stained with either Gomori's trichrome, or hematoxylin and eosin. Sections stained with hematoxylin and eosin were scored for the presence of distended ductule lumina within terminal ductal lobular unit (TDLU) structures using a subjective scale by an investigator blinded to treatment. The scoring scale reflected the number and size of ductules within TDLU that were enlarged beyond those recorded in a TDLU2 that had minimal luminal expansion. The scoring scale ranged from 1 (no expansion in any ductules) to 10 (all ductules atypically engorged). Five fields were scored for each MG, with three MG scored per pig (besides one pig that had two MG scored), excluding sections that did not contain adequate epithelial tissue (3 sections among 29).

Morphological development of epithelial TDLU structures within the pig MG was determined as we previously described and validated [14], using criteria described for the human breast [22]. Briefly, a TDLU1 in the human breast is a simple collection of approximately 11 ductules, a TDLU2 contains an average of 47 ductules, and the largest and most complex TDLU3 structures contain approximately 81 ductules [22]. We classified an average of $n=12$ TDLU (range $n=3-18$ ) in each of three whole mounts that were prepared from different regions within each of three 
MG.

Immunohistochemistry

Immunohistochemistry for phosphorylated histone H3 (PH3), phosphorylated STAT5 (PSTAT5) and progesterone receptor (PR) was performed using procedures outlined by VanKlompenberg et al. [23]. Sections of MG2, 3 and 5 (cranial to caudal) from each female were analyzed for PH3 and P-STAT5, while one section from MG3 was analyzed for PR. Each immunohistochemistry run included one section from every MG in a particular position plus a positive control section and a no primary antibody negative-control section. Blocking for PR immunohistochemistry was performed with 5\% horse serum in PBS $+0.05 \%$ Tween-20 for 30 min at room temperature. Immunoreactive PH3 was detected with rabbit anti-PH3 (1:500; Santa Cruz Biotechnology, Santa Cruz, CA) incubated at RT for $1 \mathrm{~h}$, while PR was detected using a rabbit polyclonal anti-human PR (1:100, Dako North America Inc., Carpenteria, CA) and pSTAT5 was detected using a rabbit monoclonal anti-P-STAT5 (Tyr694) antibody (1:50; Cell Signaling Technology, Danvers, MA), both overnight at $4^{\circ} \mathrm{C}$. Immunocomplexes were visualized using NovaRED chromagen (PR; Vector Laboratories, Burlingame CA) or 3,3'diaminobenzidine tetrahydrochloride (DAB; PH3 and P-STAT5; Life Technologies, Carlsbad, CA). The number of PH3, P-STAT5 or PR-positive cells and the total number of epithelial cells in each field was counted using the tag feature in Image Pro Express 6.3 (PH3; Media Cybernetics, Rockville, MD), Aperio ImageScope v10.2.2.2319 (P-STAT5; Leica Biosystems, Buffalo Grove, IL) or ImageJ (imagej.nih.gov/ij/). Quantification of cell labeling indices was performed by individuals blinded to treatment.

\section{Quantitative PCR}


Total RNA was extracted from one MG from each pig, treated with DNase and reverse transcribed as described [23]. Primers used for reference and target genes are presented in Table 1. Primers were synthesized by Sigma Life Science (St. Louis, MO) and PCR products sequence verified. Quantitative PCR reactions were performed using Fast SYBR Green master mix (Applied Biosystems, Foster City, CA), $200 \mathrm{nM}$ of each primer and $0.4 \mu \mathrm{L}$ of cDNA, in duplicate, on a 7500 Fast Real-Time PCR System (Applied Biosystems) as previously described [23] with the following modifications. The annealing and extension temperatures used for each primer set are in Table 1. Six-point relative standard curves were generated from 5-fold dilutions of either a pool of liver and MG cDNA, or MG cDNA. Mitochondrial ribosomal protein L39 and vesicle-associated membrane associated protein $\mathrm{B} / \mathrm{C}$ were used as reference genes with the following equation to normalize gene expression:

$Y_{j k}=\frac{Q_{\text {Tjk x dil } T}}{\operatorname{dilRx} \sqrt{Q_{R} 1 j k x \text { QR2jk }}}$

where $Y_{j k}$ is the normalized target gene expression for the jth female within the kth treatment, $\mathrm{Q}_{\mathrm{Tjk}}$ and $\mathrm{Q}_{\mathrm{Rjk}}$ are target and reference gene quantities, respectively, and $\operatorname{dil}_{\mathrm{T}}$ and $\operatorname{dil}_{\mathrm{R}}$ are the dilution factors for target and reference cDNA samples.

Statistical Analysis

Data were transformed to normalize variances where appropriate. Data for PH3, P-STAT5, frequency of TDLU1, TDLU2 and distended ductule scores were assessed by two-way ANOVA using a linear mixed model (SAS Institute Inc., Cary, NC). Treatment, MG position and their interaction were included as fixed effects, and pigs nested in blocks as a random effect. Data for PR labeling and relative weight gain were assessed by one-way ANOVA using a linear mixed model (SAS Institute Inc., Cary, NC). Treatment was included as a fixed effect, and pigs nested in blocks as a random effect. Normality of the residuals and presence of outliers was assessed 
using the PROC UNIVARIATE procedure in SAS by the Shapiro-Wilk test, Q-Q-plot and externally studentized residuals. Preplanned means comparisons of main effects and their interactions were performed by orthogonal contrasts in SAS. Pairwise comparisons between means or least square means were analyzed by Student's t tests. Significant effects were considered at $P \leq 0.05$ and trends at $P \leq 0.1$. All data are presented as means \pm SEM.

\section{RESULTS}

We used 6-month old cyclic pubertal female pigs in this study as a model for adolescent human females. Age at the onset of estrous was not recorded, but typically occurs around 5-months of age. Female pigs fed alcohol in blocks 1 and 2 had an average BAC of $115 \pm 4 \mathrm{mg} / \mathrm{dL}$ and $130 \pm$ $13 \mathrm{mg} / \mathrm{dL}$ (Table 2), respectively, while the control females had a BAC of $0 \mathrm{mg} / \mathrm{dL}$ for the entire study. A human $72 \mathrm{~kg}$ female is typically projected to realize a comparable BAC after binge drinking approximately 5 standard drinks within an hour, where a BAC between 60 and 150 $\mathrm{mg} / \mathrm{dL}$ in humans leads to increased impairment, while a BAC from $160 \mathrm{mg} / \mathrm{dL}$ to $300 \mathrm{mg} / \mathrm{dL}$ leads to severe impairment [24]. Blood alcohol concentrations $<85 \mathrm{mg} / \mathrm{dL}$ were associated with less than complete meal consumption within the preceding 24 h. Females in block 1 gained 26.5 $\mathrm{kg}$ (control) and $25 \mathrm{~kg}$ (alcohol) over the 36-day experimental period. The control females in block 2 gained $7.5 \pm 2.0 \mathrm{~kg}$ during the 46-47d experimental period while those consuming alcohol gained an average of $2.9 \pm 2.9 \mathrm{~kg}$, with no difference between treatments (Figure 1; $\mathrm{P}=0.23)$.

The weight of the heart, kidneys and uterus was not different for the females fed either the control or alcohol-supplemented diets (Table 3; P>0.1). By contrast, the livers from alcohol-fed females were heavier than those from the control females (Table 3; $\mathrm{P}<0.05$ ). 
We next analyzed histomorphogenesis within the MG from control and alcohol-fed females, where classification of structures as different TDLU morphotypes reflects the complexity of lobular branching [22]. Consistent with our previous findings [14], the epithelial structures in the MG of control-fed pubertal female pigs included TDLU types 1-3, with a predominance of type 2. After pigs were fed the alcohol-supplemented diet their MG had an increased proportion of TDLU2 ( $\mathrm{P}=0.02)$ that was accompanied by a corresponding decrease in the incidence of TDLU1 $(\mathrm{P}=0.02)$. There was a significant effect of $\mathrm{MG}$ position on both TDLU1 frequency $(\mathrm{P}=0.02)$ and TDLU2 frequency $(\mathrm{P}=0.02)$ but no interactions between the effect of alcohol and MG position ( $\mathrm{P}=0.71$ and $\mathrm{P}=0.62$ respectively). Specifically, the frequency of TDLU1 was lower in MG5 versus MG2 ( $\mathrm{P}=0.005)$ and correspondingly, the frequency of TDLU2 was higher in MG5 versus MG2 ( $\mathrm{P}=0.006)$. The low incidence of TDLU3 in all the glands analyzed precluded any analysis of a position effect.

Further analysis of the MG parenchyma revealed that the histomorphogenesis of these TDLU was also altered in response to alcohol supplementation. Specifically, alcohol induced dilation of ductular lumina within TDLU ( $\mathrm{P}=0.02$; Figure $2 \mathrm{~F})$. There was no effect of MG position on this phenotype $(P=0.78)$ and no interaction between $M G$ position and treatment $(P=0.48)$. Trichrome staining revealed that these alcohol-induced changes were associated with increased expansion of the collagenous intralobular stroma (Figure 2D), and increased accumulation of material, presumed to be proteinaceous, within distended ductular lumina (Figure 2D).

We investigated whether dilation of the ductules in response to alcohol and/or progression of the TDLU1 structures toward a TDLU2 morphotype was associated with increased epithelial proliferation. Proliferation of epithelial cells as measured by immunohistochemistry for PH3 was increased in the MG of females fed alcohol compared to controls (Figure 3; $\mathrm{P}<0.05$ ). There was 
no overall effect of $\mathrm{MG}$ position $(\mathrm{P}=0.95)$ and no interaction between $\mathrm{MG}$ position and treatment $(\mathrm{P}=0.80)$ on this measure.

Given that activation of STAT5 by phosphorylation is associated with both epithelial proliferation and lactogenic differentiation in the MG [25, 26], we examined its abundance after alcohol supplementation. Immunostaining for P-STAT5 was increased in the MG of females fed alcohol (Figure 4; $\mathrm{P}<0.05)$. There was no effect of $\mathrm{MG}$ position $(\mathrm{P}=0.62)$ and no interaction between $\mathrm{MG}$ position and treatment $(\mathrm{P}=0.97)$ for this variable.

In a previous study we recorded that epithelial proliferation was induced synergistically by exogenous estrogen and prolactin [14]. Based on our unpublished microarray data, we examined whether the expression of genes that were up- or down-regulated by the combination of these hormones (SLC28A3, AK345252, AK235587, TNXB, ATP6V1C2 and c-jun) was also affected by alcohol consumption. As shown in Figure 5, the expression of these genes in the MG of alcohol-fed pigs was not different to that in control-fed pigs ( $\mathrm{P}>0.1$; Figure 5 ). Given that the increased phosphorylation of STAT5, distended ductules and proteinaceous material in the lumina of females fed alcohol may have reflected precocious lactogenesis, we examined expression of prolactin responsive and lactation-associated genes (ß-casein, $\alpha$-lactalbumin, LPL, and FcRn). No difference in gene expression for any of these targets was detected ( $\mathrm{P}>0.1$; Figure 5). Furthermore, neither IGF-I nor IGFBP3 mRNA levels in the MG were affected by alcohol intake ( $\mathrm{P}>0.1$; Figure 5) despite their associations with IGF action and breast density in humans [27].

We also analyzed the incidence and localization of epithelial PR (both A and B isoforms) in response to supplemental alcohol given its prognostic significance and sensitivity to estrogenic 
stimulation. As depicted in Figure 6, there was no difference in the proportion of mammary epithelial cells expressing PR in the MG of control and alcohol-treated females ( $\mathrm{P}>0.9)$.

\section{DISCUSSION}

The histoarchitecture within the human breast includes epithelial structures arranged as complex TDLU [22] that grow within a unique stromal microenvironment consisting of both dense fibroblastic interlobular stroma, and loosely connected intralobular stroma [28]. Given that alcohol consumption leads to a dose-dependent increase in breast cancer risk in humans, and increases the incidence of benign breast disease during adolescence [3], a pressing question is how alcohol intake predisposes the normal human breast to future cancer. Modeling these changes in an animal model that recapitulates aspects of both breast development and alcohol consumption stands to provide important insights to the mechanism(s) by which voluntary alcohol consumption by women increases breast cancer risk throughout life.

A short period of excessive alcohol intake induced local changes in the MG of pigs consistent with alterations that might correspond to increased risk for subsequent breast disease in the presence of one or more transforming events. Specifically, we identified increased proliferation of the mammary epithelium alongside increased phosphorylation of STAT5. Others have shown that STAT5 activation increases cell proliferation and promotes tumor formation [29, 30] by activating mitogen-activated protein kinases and Akt [31, 32], and by regulating the expression of phosphatidylinositol 3-kinase to increase cell survival [30]. The role of these and other downstream changes in response to the effects of alcohol in this model remain to be established. At the same time we did not record any evidence of lactogenic differentiation at the level of transcriptional changes for several milk proteins, despite dilation of the lobular ductules and the accumulation of proteinaceous material in the lumina. Given these data, we propose that alcohol- 
induced phosphorylation of STAT5 associates with enhanced mitogenic signaling rather than precocious lactogenesis.

Our finding that proliferation of the mammary epithelium of pigs was increased by alcohol intake aligns with data indicating that mammary epithelial growth was also increased in alcoholfed mice and rats. When pubertal mice were fed low concentrations (3.2\%) of alcohol via the drinking water there was increased ductal branching in the MG [33]. Similar changes were recorded in the MG of rats fed higher concentrations of ethanol [34]. As we and other authors have continually highlighted, the human breast undergoes a histomorphogenesis that is much more complex than is seen in rodents, but which is more closely recapitulated by the MG of pigs [14, 35]. The progression of TDLU type, as was promoted by alcohol herein, is responsive to endocrine changes in humans and pigs [22] [14]. The fact that alcohol increased epithelial proliferation and progression of TDLU type in the MG of pigs aligns well with the increased mammographic density that occurs in humans as a result of alcohol intake [36], which itself predicts breast cancer risk [37]. Thus, an animal model such as the pig that precisely defines proliferative changes within the complex TDLU morphotypes and the associated unique stromal elements stands to provide important insights to understanding the relationship between alcohol intake and breast cancer risk.

The mechanism by which alcohol increases epithelial proliferation remains to be resolved. As in humans, alcohol intake by pigs increased hepatic mass, which over longer periods leads to hepatic cirrhosis in this species [38]. One potential consequence of altered hepatic function is reduced clearance of circulating steroid hormones, such as was found for postmenopausal women administered exogenous estrogen [39] which, in turn, would lead to increased stimulation of the mammary epithelium. In the present study we also analyzed PR expression within the MG 
as a potential marker of increased estrogen activation in response to alcohol intake, but did not detect any changes at that level. Alcohol intake also causes hyperprolactinemia in humans, nonhuman primates and laboratory animals $[40,41]$ and high levels of prolactin are associated with an increased risk of breast cancer, particularly estrogen receptor-positive tumors [42]. Prolactin is also a potent activator of STAT5 [25]. Previously we found that the actions of prolactin and estrogen interact to stimulate the proliferation of mammary epithelial cells and TDLU formation [14]. Whether estrogen and prolactin signalling converge in response to alcohol intake remains to be confirmed.

Alcohol intake may also increase breast cancer risk via impaired one-carbon metabolism in association with folate deficiency, leading to increased DNA damage and/or reduced DNA repair [43]. Alternatively, alcohol-induced changes in methylation can result in an increased sensitivity to carcinogens [44], generation of mutagens such as acetaldehyde and reactive oxygen species, or perturbations in estrogen signaling [43]. Future investigation into whether these or other mechanisms are at play in pigs remains to occur.

In conclusion, these studies highlight that consumption of alcohol by pigs leads to proliferative changes in the MG consistent with its ability to increase breast cancer risk in humans. Given the broad similarities between the MG of pigs and humans, as well as their genetic and metabolic similarities, we propose that this model stands to provide further opportunities to determine how alcohol consumption by humans increases breast cancer risk.

\section{ACKNOWLEDGMENTS}

We thank Hatley McMicking, Brittany Bazeley, Rachel de Jesus, Kent Parker and interns for assistance with animal work, Caleb Sehnert for assistance with collection of tissues, Richard Duplantier and John Frey for analyzing BAC and Dr Charles Halsted for helpful suggestions 
regarding study design. We are also grateful to Dr Bob Cardiff, Dr Sandy Borowsky and Dr Graham Colditz for helpful suggestions and guidance regarding this work. MVK was supported by NSF National Science Foundation Graduate Research Fellowship DGE-0707429.

\section{BIBLIOGRAPHY}

[1] Williams RR, Horm JW. Association of cancer sites with tobacco and alcohol consumption and socioeconomic status of patients: interview study from the Third National Cancer Survey. J Natl Cancer Inst. 1977;58:525-47.

[2] Allen NE, Beral V, Casabonne D, Kan SW, Reeves GK, Brown A, et al. Moderate alcohol intake and cancer incidence in women. J Natl Cancer Inst. 2009;101:296-305.

[3] Berkey CS, Willett WC, Frazier AL, Rosner B, Tamimi RM, Rockett HR, et al. Prospective study of adolescent alcohol consumption and risk of benign breast disease in young women. Pediatrics. 2010;125:1081-7.

[4] Hamajima N, Hirose K, Tajima K, Rohan T, Calle EE, Heath CW, Jr., et al. Alcohol, tobacco and breast cancer--collaborative reanalysis of individual data from 53 epidemiological studies, including 58,515 women with breast cancer and 95,067 women without the disease. Br J Cancer. 2002;87:1234-45.

[5] Key J, Hodgson S, Omar RZ, Jensen TK, Thompson SG, Boobis AR, et al. Meta-analysis of studies of alcohol and breast cancer with consideration of the methodological issues. Cancer Causes Control. 2006;17:759-70.

[6] Lew JQ, Freedman ND, Leitzmann MF, Brinton LA, Hoover RN, Hollenbeck AR, et al. Alcohol and risk of breast cancer by histologic type and hormone receptor status in postmenopausal women: the NIH-AARP Diet and Health Study. Am J Epidemiol. 2009;170:308-17. 
[7] Breast Cancer Risk in American Women. National Cancer Institute; 2012.

[8] National Institute on Alcohol Abuse and Alcoholism.

NIAAA Council Approves Definition of Binge Drinking. In: NIAAA Newsletter, Roa G editor.

Vol 3. http://pubs.niaaa.nih.gov/publications/Newsletter/winter2004/Newsletter_Number3.pdf:

Office of Research Translation and Communications NIAAA NIH DHHS; 2004. p. 3.

[9] Kanny D, Liu Y, Brewer RD, Garvin W, Balluz L. Vital Signs: Binge Drinking Among High

School Students and Adults — United States, 2009. Morb Mortal Weekly Rep: Centers for

Disease Control and Prevention; 2010.

[10] Byrne C, Webb PM, Jacobs TW, Peiro G, Schnitt SJ, Connolly JL, et al. Alcohol consumption and incidence of benign breast disease. Cancer Epidemiol Biomarkers Prev. 2002;11:1369-74.

[11] Hovey RC, Trott JF, Vonderhaar BK. Establishing a framework for the functional mammary gland: from endocrinology to morphology. J Mammary Gland Biol Neoplasia. 2002;7:17-38.

[12] Land CE. Studies of cancer and radiation dose among atomic bomb survivors. The example of breast cancer. JAMA. 1995;274:402-7.

[13] Russo J, Hu YF, Yang X, Russo IH. Developmental, cellular, and molecular basis of human breast cancer. J Natl Cancer Inst Monogr. 2000:17-37.

[14] Horigan KC, Trott JF, Barndollar AS, Scudder JM, Blauwiekel RM, Hovey RC. Hormone interactions confer specific proliferative and histomorphogenic responses in the porcine mammary gland. Domest Anim Endocrinol. 2009;37:124-38.

[15] Rowson AR, Daniels KM, Ellis SE, Hovey RC. Growth and development of the mammary glands of livestock: a veritable barnyard of opportunities. Semin Cell Dev Biol. 2012;23:557-66. 
[16] Wernersson R, Schierup MH, Jorgensen FG, Gorodkin J, Panitz F, Staerfeldt HH, et al. Pigs in sequence space: a 0.66X coverage pig genome survey based on shotgun sequencing. BMC Genomics. 2005;6:70.

[17] Swindle MM, Makin A, Herron AJ, Clubb FJ, Jr., Frazier KS. Swine as models in biomedical research and toxicology testing. Vet Pathol. 2012;49:344-56.

[18] Dexter JD, Tumbleson ME, Decker JD, Middleton CC. Comparison of the offspring of three serial pregnancies during voluntary alcohol consumption in Sinclair (S-1) miniature swine. Neurobehav Toxicol Teratol. 1983;5:229-31.

[19] Rumpler WV, Rhodes DG, Baer DJ, Conway JM, Seale JL. Energy value of moderate alcohol consumption by humans. Am J Clin Nutr. 1996;64:108-14.

[20] Livesey G. Metabolizable energy of macronutrients. Am J Clin Nutr. 1995;62:1135S-42S. [21] Hovey RC, Auldist DE, Mackenzie DD, McFadden TB. Preparation of an epithelium-free mammary fat pad and subsequent mammogenesis in ewes. J Anim Sci. 2000;78:2177-85. [22] Russo J, Hu YF, Silva IDCG, Russo IH. Cancer risk related to mammary gland structure and development. Microsc Res Tech. 2001;52:204-23.

[23] Vanklompenberg MK, Manjarin R, Trott JF, McMicking HF, Hovey RC. Late gestational hyperprolactinemia accelerates mammary epithelial cell differentiation that leads to increased milk yield. J Anim Sci. 2013;91:1102-11.

[24] National Institute on Alcohol Abuse and Alcoholism. Alcohol Overdose: The Dangers of Drinking Too Much.

http://pubsniaaanihgov/publications/AlcoholOverdoseFactsheet/Overdosefacthtm: National Institutes of Health; 2013. p. 1-3. 
[25] Hennighausen L, Robinson GW, Wagner KU, Liu X. Developing a mammary gland is a Stat affair. J Mammary Gland Biol Neoplasia. 1997;2:365-72.

[26] Miyoshi K, Shillingford JM, Smith GH, Grimm SL, Wagner KU, Oka T, et al. Signal transducer and activator of transcription (Stat) 5 controls the proliferation and differentiation of mammary alveolar epithelium. J Cell Biol. 2001;155:531-42.

[27] Diorio C, Pollak M, Byrne C, Masse B, Hebert-Croteau N, Yaffe M, et al. Insulin-like growth factor-I, IGF-binding protein-3, and mammographic breast density. Cancer Epidemiol Biomarkers Prev. 2005;14:1065-73.

[28] Russo J, Russo IH. Development of the human mammary gland. In: Neville MC, Daniel CW, editors. The mammary gland Development, regulation and function. New York: Plenum; 1987. p. 67-93.

[29] Buettner R, Mora LB, Jove R. Activated STAT signaling in human tumors provides novel molecular targets for therapeutic intervention. Clin Cancer Res. 2002;8:945-54.

[30] Schmidt JW, Wehde BL, Sakamoto K, Triplett AA, Anderson SM, Tsichlis PN, et al. Stat5 regulates the phosphatidylinositol 3-kinase/Akt1 pathway during mammary gland development and tumorigenesis. Mol Cell Biol. 2014;34:1363-77.

[31] Wu Y, Kim J, Elshimali Y, Sarkissyan M, Vadgama JV. Activation of Akt1 accelerates carcinogen-induced tumorigenesis in mammary gland of virgin and post-lactating transgenic mice. BMC Cancer. 2014;14:266.

[32] Nyga R, Pecquet C, Harir N, Gu H, Dhennin-Duthille I, Regnier A, et al. Activated STAT5 proteins induce activation of the PI 3-kinase/Akt and Ras/MAPK pathways via the Gab2 scaffolding adapter. Biochem J. 2005;390:359-66. 
[33] Masso-Welch PA, Tobias ME, Vasantha Kumar SC, Bodziak M, Mashtare T, Jr., Tamburlin J, et al. Folate exacerbates the effects of ethanol on peripubertal mouse mammary gland development. Alcohol. 2012;46:285-92.

[34] Singletary KW, McNary MQ. Influence of ethanol intake on mammary gland morphology and cell proliferation in normal and carcinogen-treated rats. Alcohol Clin Exp Res. 1994;18:1261-6.

[35] Howard BA, Gusterson BA. Human breast development. J Mammary Gland Biol Neoplasia. 2000;5:119-37.

[36] Flom JD, Ferris JS, Tehranifar P, Terry MB. Alcohol intake over the life course and mammographic density. Breast Cancer Res Treat. 2009;117:643-51.

[37] McCormack VA, dos Santos Silva I. Breast density and parenchymal patterns as markers of breast cancer risk: a meta-analysis. Cancer Epidemiol Biomarkers Prev. 2006;15:1159-69. [38] Niemela O, Parkkila S, Yla-Herttuala S, Villanueva J, Ruebner B, Halsted CH. Sequential acetaldehyde production, lipid peroxidation, and fibrogenesis in micropig model of alcoholinduced liver disease. Hepatology. 1995;22:1208-14.

[39] Ginsburg ES, Walsh BW, Shea BF, Gao X, Gleason RE, Barbieri RL. The effects of ethanol on the clearance of estradiol in postmenopausal women. Fertil Steril. 1995;63:1227-30. [40] De A, Boyadjieva N, Oomizu S, Sarkar DK. Ethanol induces hyperprolactinemia by increasing prolactin release and lactotrope growth in female rats. Alcohol Clin Exp Res. 2002;26:1420-9.

[41] Sarkar DK. Hyperprolactinemia following chronic alcohol administration. Front Horm Res. 2010;38:32-41. 
[42] Tworoger SS, Hankinson SE. Prolactin and breast cancer etiology: an epidemiologic perspective. J Mammary Gland Biol Neoplasia. 2008;13:41-53.

[43] Dumitrescu RG, Shields PG. The etiology of alcohol-induced breast cancer. Alcohol. 2005;35:213-25.

[44] Christensen BC, Kelsey KT, Zheng S, Houseman EA, Marsit CJ, Wrensch MR, et al. Breast cancer DNA methylation profiles are associated with tumor size and alcohol and folate intake.

PLoS Genet. 2010;6:e1001043. 


\section{FIGURE LEGENDS}

Figure 1. Total weight gain by pigs over the experimental period as a percentage of initial weight. Females were fed either the control diet or alcohol-supplemented diet. Data are means \pm SEM ( $\mathrm{n}=5)$. Bodyweights were not different between treatments $(\mathrm{P}=0.4)$.

Figure 2. Alcohol intake increases the percentage of terminal ductal lobular units type 2 (TDLU2) in the mammary glands (MG) alongside distension of the ductular lumina. A and B) Representative images from carmine alum stained whole mounts from control (A) and alcoholfed (B) female pigs. A representative TDLU2 is circled in each panel. Scale bar $=250 \mu \mathrm{m}$. A) Black arrow points to a normal ductule. B) Black arrow points to a distended ductule. C and D) Gomori's trichrome-stained sections of a TDLU2 from control (A) and alcohol-fed (B) female pigs. Scale bar $=50 \mu \mathrm{m}$. Trichrome stains nuclei blue/black, collagen blue and cytoplasm, muscle and plasma proteins red. C) Arrow points to a normal ductule. Arrowhead points to intralobular stroma. D) Arrow points to a dilated ductule having eosinophilic secretion within its lumen. Arrowhead points to expanded intralobular stroma. E) The frequency of TDLU types (1, 2 and 3) within the MG of each female was determined from wholemounts. Data are means \pm SEM ( $\mathrm{n}=5) .{ }^{*} \mathrm{P}<0.05$ for a treatment effect within a TDLU type. F) The incidence of distended ductules was scored from hematoxylin and eosin stained sections using a subjective scale of 1-10 (see materials and methods). Data are means $\pm \mathrm{SEM}(\mathrm{n}=5) . * \mathrm{P}<0.05$.

Figure 3. Epithelial cell proliferation was increased in the mammary glands (MG) of alcohol-fed female pigs. Paraffin sections of MG were immunostained for phosphorylated histone H3 (PH3) using DAB chromogen, then counterstained with hematoxylin. Representative sections for PH3 
staining in the MG from A) control and B) alcohol fed pigs. Scale bar $=50 \mu \mathrm{m}$. C) The incidence of PH3-positive cells was quantified as a percentage of the total number of epithelial cells. At least 400 epithelial cells were counted across five independent fields in each of three MG/pig. Data are the frequency of positive cells/MG \pm SEM $(\mathrm{n}=5) . * \mathrm{P}<0.05$.

Figure 4. Phosphorylated signal transducer and activator of transcription (pSTAT)-5 in the mammary glands (MG) of pigs is increased by alcohol intake. Paraffin sections of MG were immunostained for pSTAT5 using DAB chromogen, then were counterstained with hematoxylin. Representative sections of MG from A) control and B) alcohol fed female pigs. C) The incidence of cells that was positive for pSTAT-5 was quantified as a percentage of total epithelial cells (range of 3415- 49296 cells per section) in each MG for three MG/pig. Scale bar = $100 \mu \mathrm{m}$. Data are the frequency of positive cells/MG $\pm \operatorname{SEM}(\mathrm{n}=5)$. $* \mathrm{P}<0.05$.

Figure 5. Relative gene expression did not differ in the mammary glands (MG) of control and alcohol fed female pigs. Relative expression of mRNA for AK235587, AK345252, $\beta$-casein, insulin like growth factor-I (IGF-I), IGF binding protein 3 (IGFBP3), neonatal Fc receptor (FcRn), $\alpha$-lactalbumin, lipoprotein lipase (LPL), ATPase (ATP6V1C2), c-jun, solute carrier family 35 member B2 (SLC35B2) and tenascin (TNXB) was determined by reverse transcription- quantitative PCR of RNA extracted from the MG ( $\mathrm{n}=1 \mathrm{MG} / \mathrm{pig}$ ). Data are means \pm $\operatorname{SEM}(n=4-5)$.

Figure 6. Progesterone receptor expression was unchanged in the mammary glands (MG) of alcohol-fed female pigs. Paraffin sections of MG were immunostained for progesterone receptor 
(PR) using NovaRED chromogen with hematoxylin counterstain. A) Representative section from an alcohol-fed female and B) negative control section incubated with biotinylated donkey antirabbit secondary antibody. C) Positive nuclei were quantified as a percentage of total cells. More than 200 epithelial cells/field from four independent fields were counted for each section from 1 MG per pig. Scale bar $=50 \mu \mathrm{m}$. Data are the average frequency for PR-positive cells $/ \mathrm{MG} \pm \mathrm{SEM}$ $(n=5)$. 
Table 2. Blood alcohol concentration (BAC) of female pigs in block 1 fed alcohol starting on day 3 , and pigs in block 2 fed alcohol starting on day 4 . The concentration of alcohol in the feed was unchanged from day 8 - 35 for pigs in block 1 , and from day 12 - day 46 for pigs in block 2 . All control pigs registered a BAC of zero.

\begin{tabular}{|l|l|}
\cline { 2 - 2 } \multicolumn{1}{|c|}{ Block 1 } & BAC (mg/dL) \\
\hline Day & Alcohol-fed female \\
\hline 17 & 119.5 \\
\hline 35 & 111 \\
\hline
\end{tabular}

\begin{tabular}{|l|l|l|l|l|}
\cline { 2 - 5 } \multicolumn{1}{c|}{ Block 2 } & \multicolumn{4}{c|}{ BAC (mg/dL) } \\
\hline Day & $\mathbf{1 9 - 1}$ & $\mathbf{1 9 - 5}$ & $\mathbf{2 2 - 1}$ & $\mathbf{2 1 - 3}$ \\
\hline 10 & 293.7 & 24 & 288.1 & 290.4 \\
\hline 12 & 309.4 & 95 & 326.7 & 81.3 \\
\hline 14 & 52 & 88 & 0 & 68 \\
\hline 17 & 139 & 160 & 204 & 125 \\
\hline 24 & 83 & 131 & 153 & 191 \\
\hline 31 & 33 & 97 & 0 & 136 \\
\hline 33 & 183 & 127 & 125 & 75 \\
\hline 38 & 190 & 140 & 151 & 93 \\
\hline 46 & 23 & 0 & 135 & 76 \\
\hline
\end{tabular}


Table 3. Organ masses, corrected for metabolic body weight (MBW) harvested at necropsy from pigs fed the control or alcohol-supplemented diets. Data are means \pm SEM $(n=5) .{ }^{*} \mathrm{P}<0.05$ for alcohol vs control.

\begin{tabular}{|l|l|l|}
\cline { 2 - 3 } \multicolumn{1}{c|}{} & \multicolumn{2}{l|}{ Weight/MBW (g/kg) } \\
\cline { 2 - 3 } \multicolumn{1}{c|}{} & Control & Alcohol \\
\hline Liver & $45.8 \pm 1.3$ & $60.5^{*} \pm 6.4$ \\
\hline Kidneys & $10.0 \pm 0.3$ & $10.8 \pm 0.3$ \\
\hline Uterus & $20.6 \pm 1.7$ & $21.7 \pm 2.4$ \\
\hline Heart & $10.2 \pm 0.3$ & $10.9 \pm 0.2$ \\
\hline
\end{tabular}




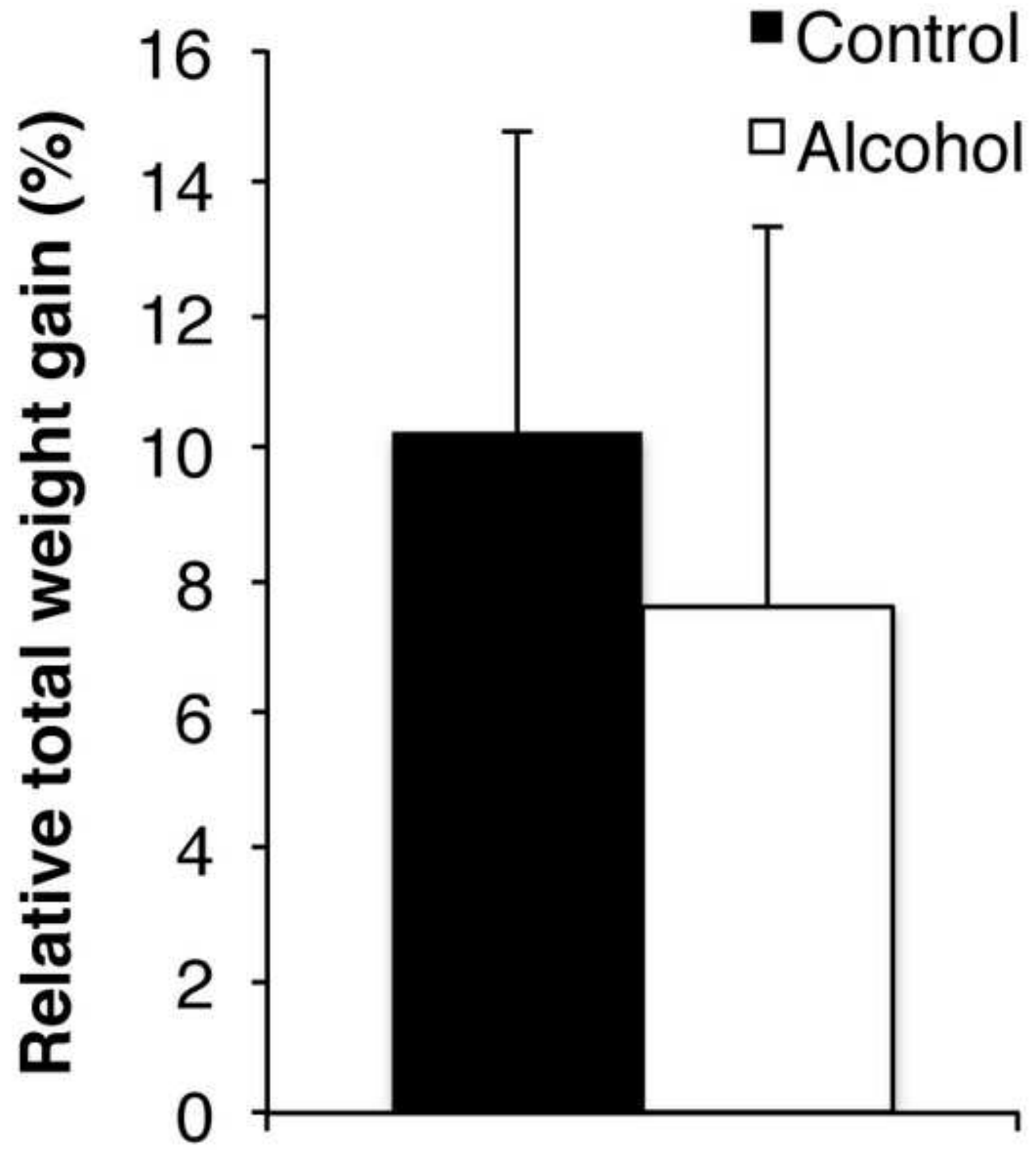

\section{- Control}

$\square$ Alcohol

등

둔

용

(4)

3

홍

9

$\geq$

힘

ब)

ฯ 
Control
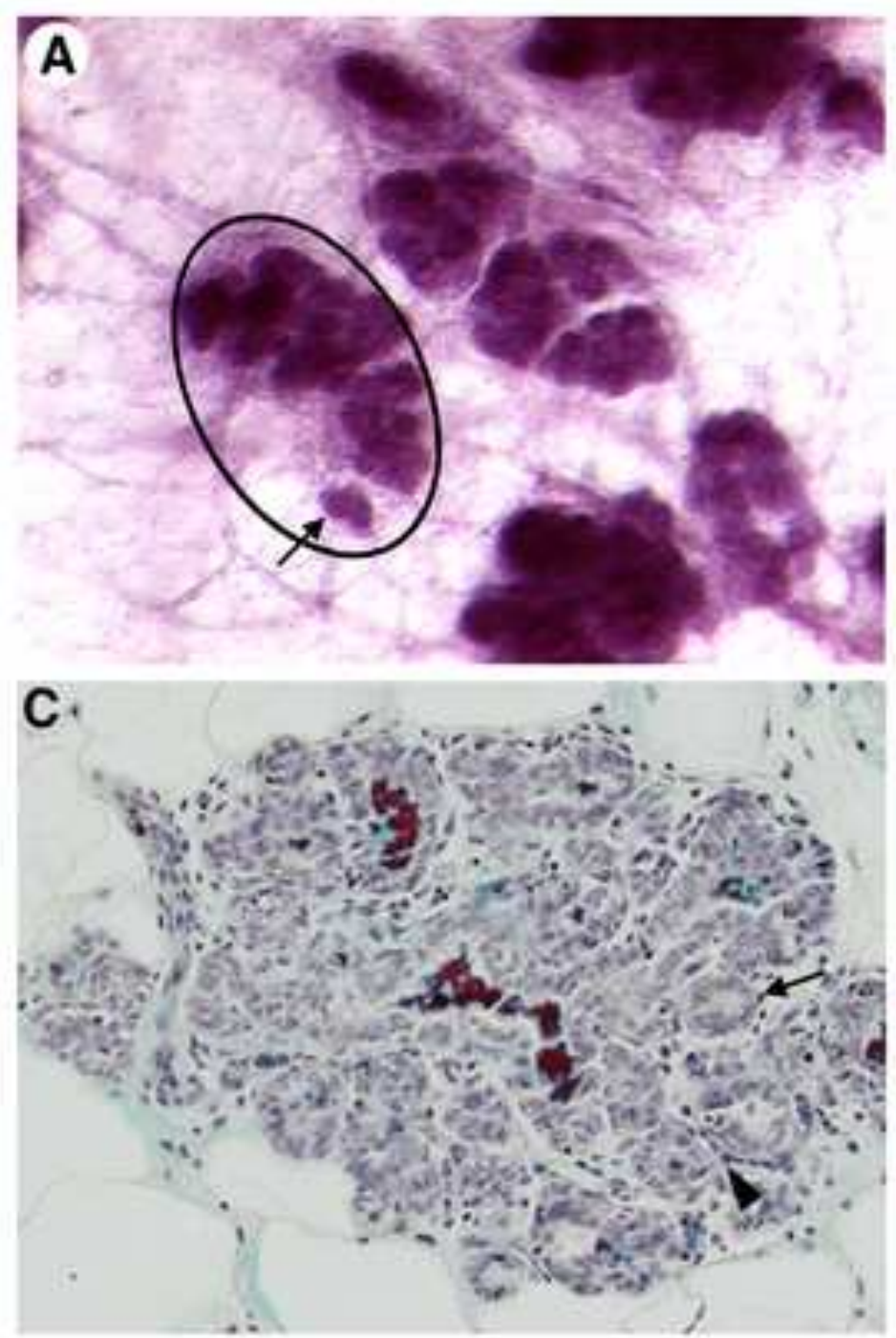

E

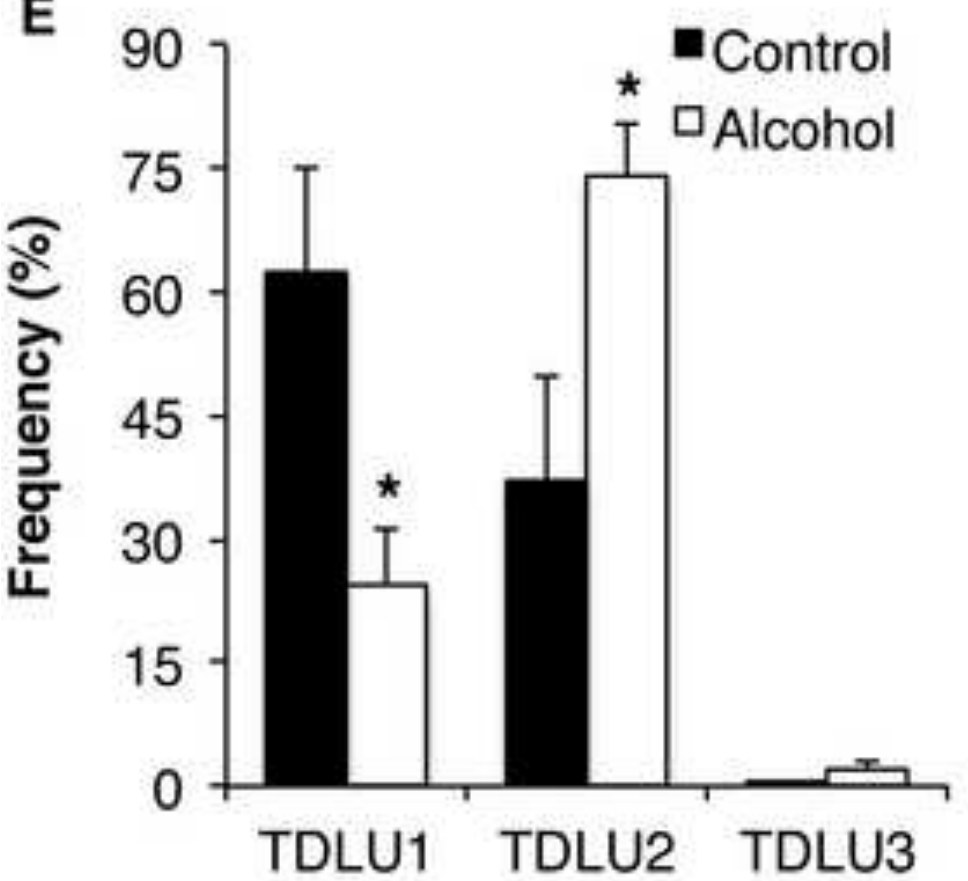

Alcohol
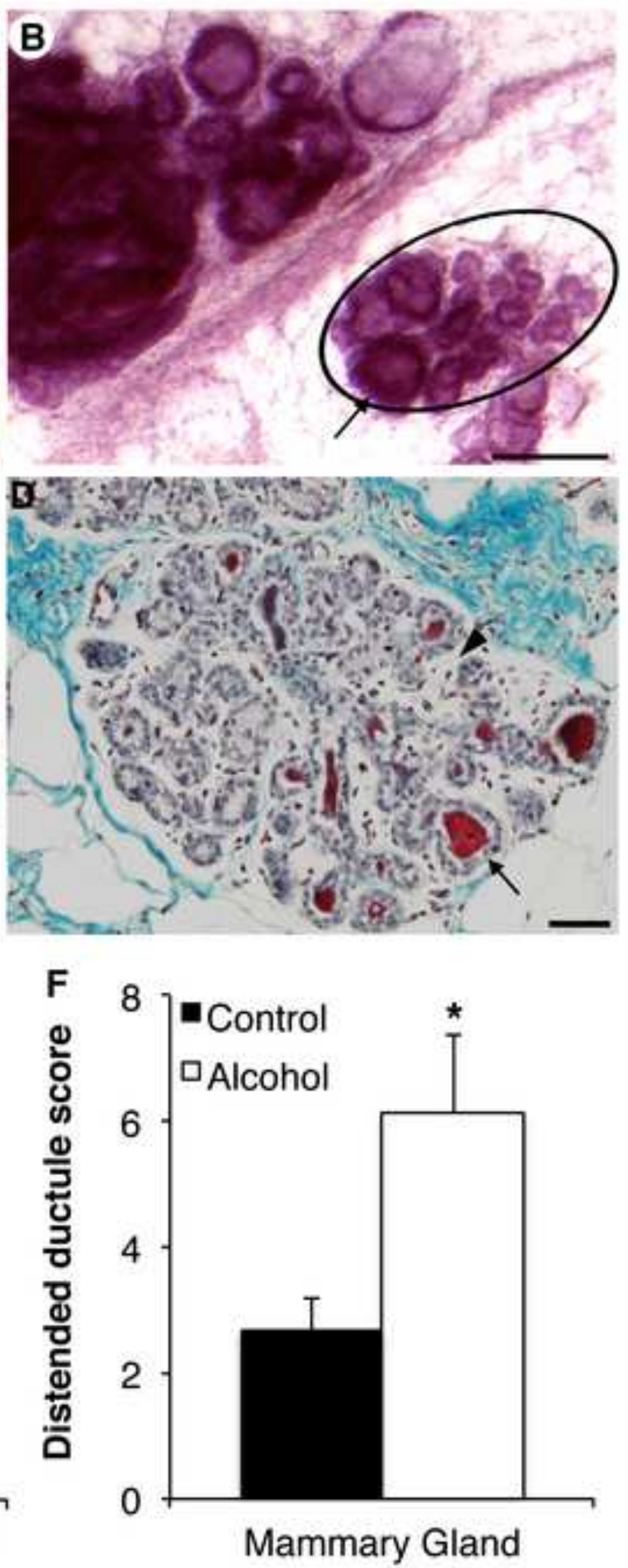

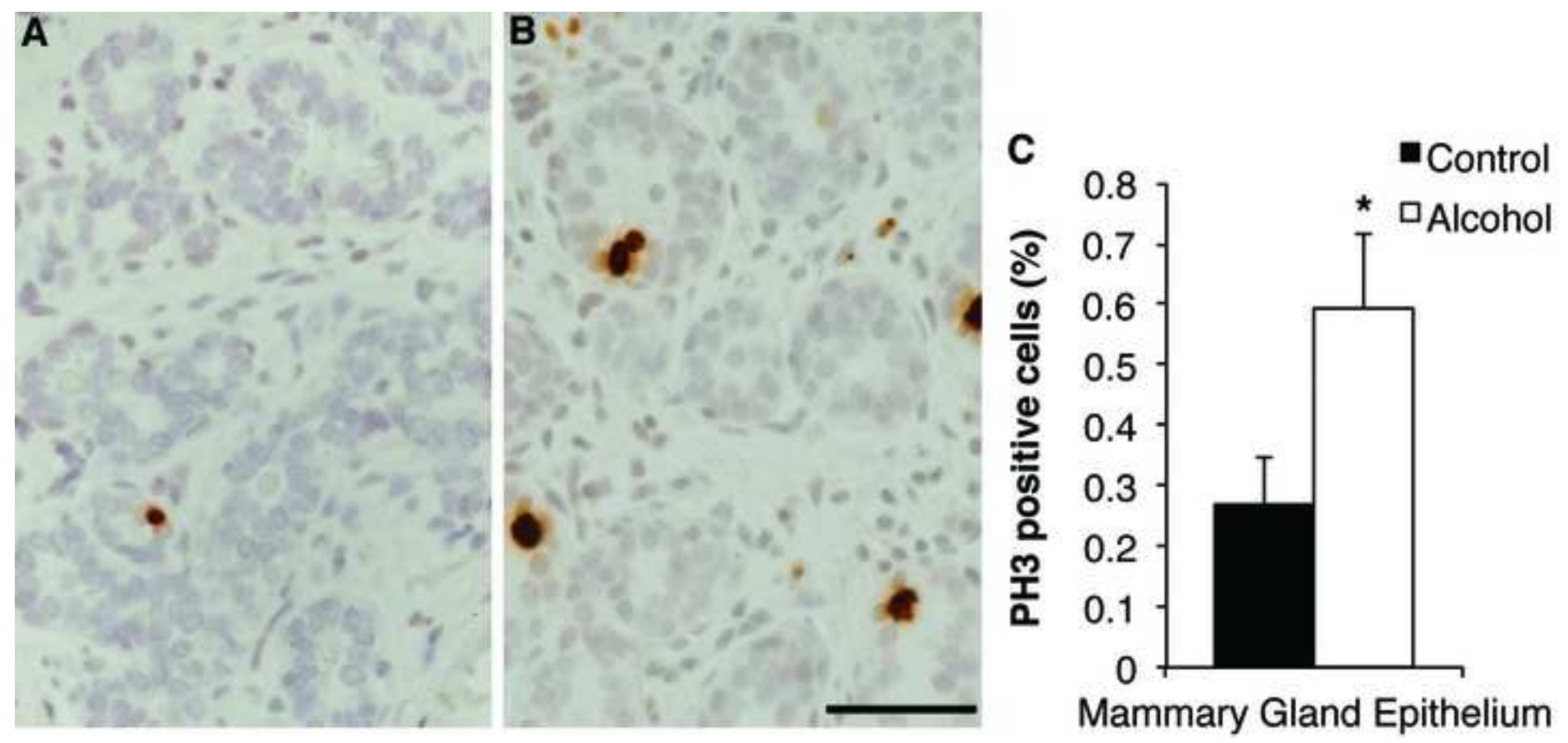

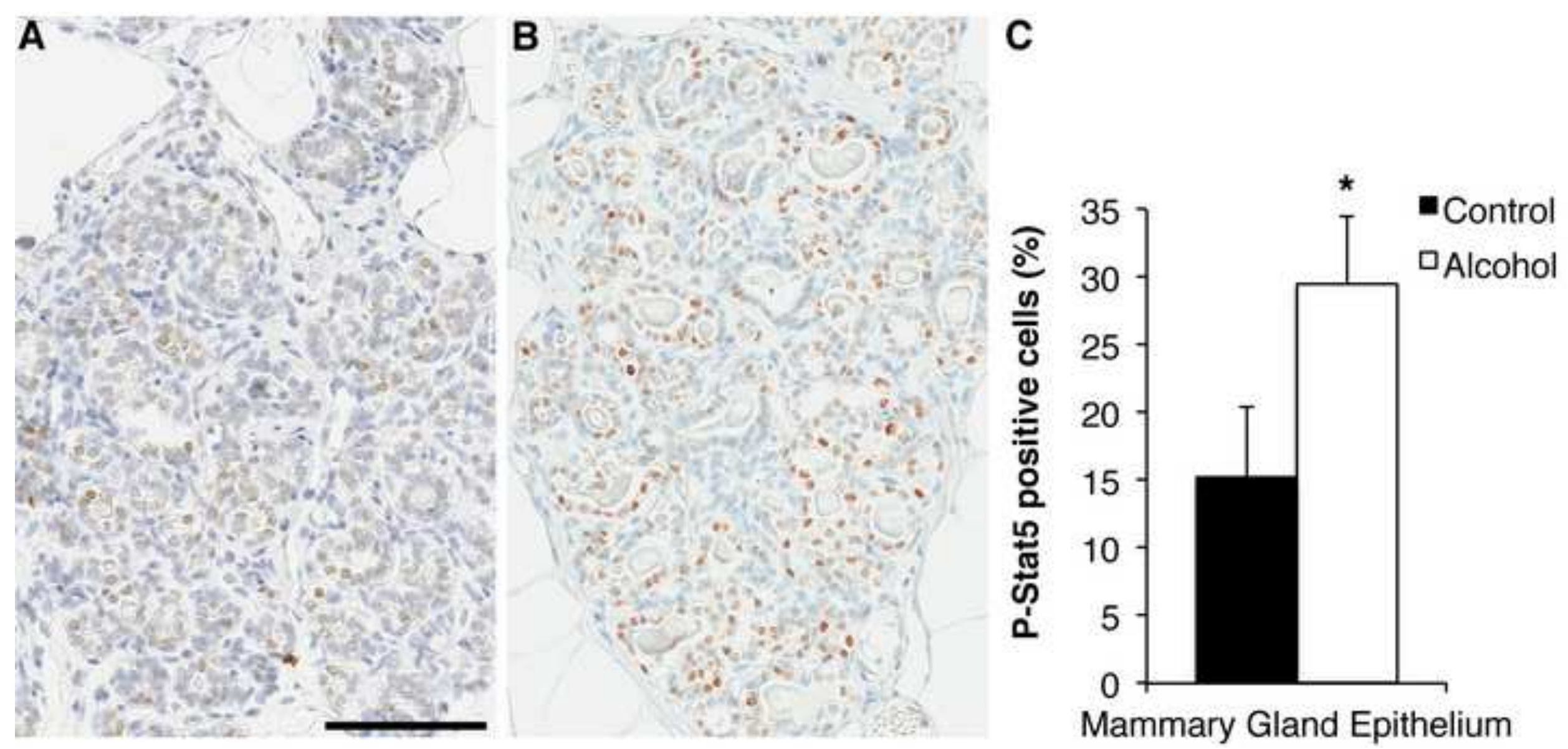


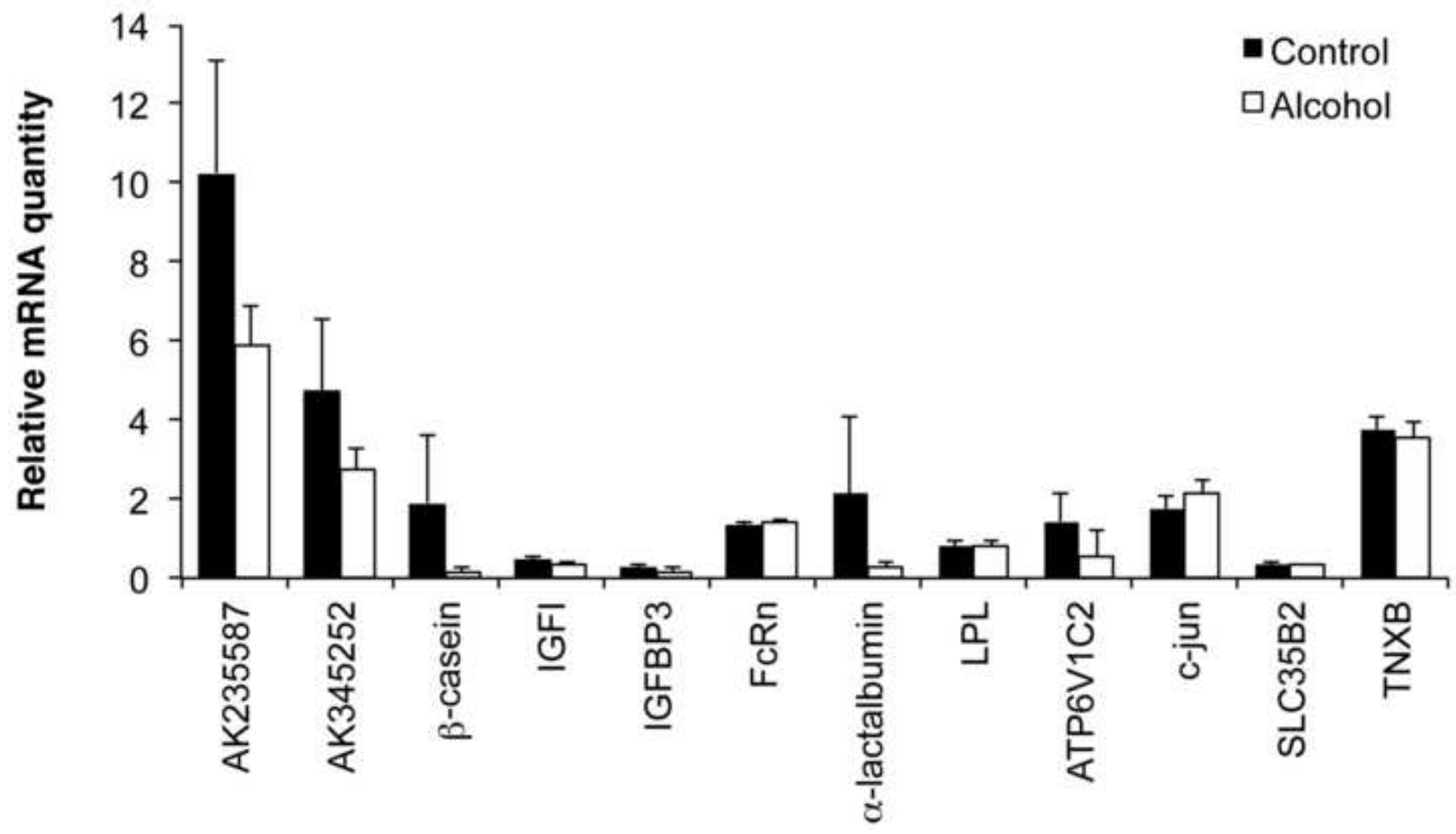



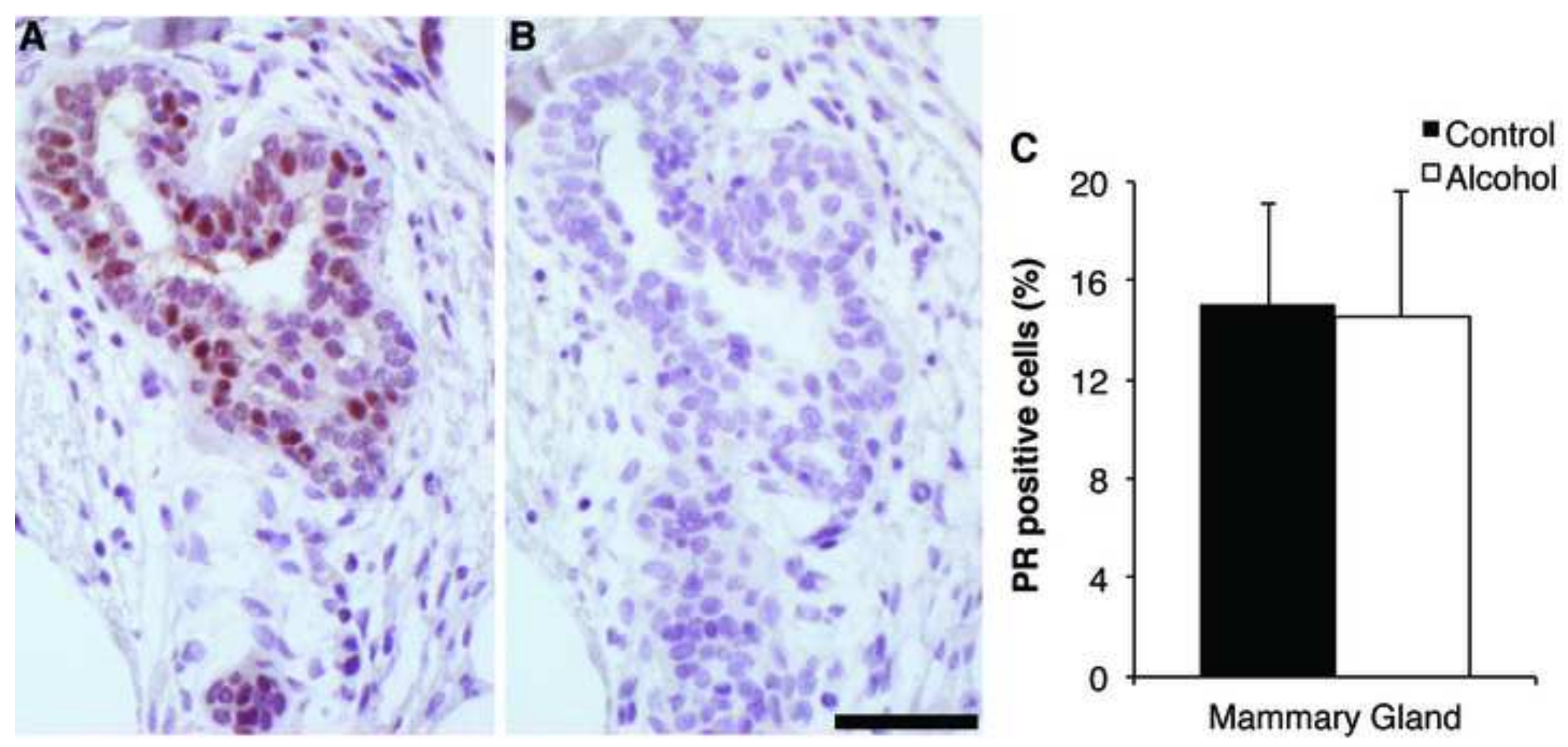